\title{
Increased local expressions of CX3CLI and CCL2 are related to clinical severity in lumbar disk herniation patients with sciatic pain
}

This article was published in the following Dove Press journal: Journal of Pain Research

17 January 2017

Number of times this article has been viewed

\author{
Zhen-Yu Peng ${ }^{1,2}$ \\ Rui Chen ${ }^{3}$ \\ Zuo-Zhong Fang ${ }^{2}$ \\ Bin Chen² \\ Zhi-Hua Wang ${ }^{4}$ \\ Xi-Yang Wang' \\ 'Department of Spine Surgery, \\ Xiangya Hospital, Central South \\ University, Changsha, ${ }^{2}$ Department \\ of Spine Surgery, Chenzhou No.I \\ People's Hospital, Chenzhou, \\ ${ }^{3}$ Department of Neurosurgery, \\ Nanhua Hospital Affiliated to Nanhua \\ University, Hengyang, ${ }^{4}$ Department of \\ Orthopedics, Chenzhou No.I People's \\ Hospital, Chenzhou, Hunan, People's \\ Republic of China
}

Background: Chemokines have been identified to be involved in the modulation of pain through both peripheral and central mechanisms. However, the role of chemokines in lumbar disk herniation $(\mathrm{LDH})$ with sciatic pain remains unknown.

Objective: The current study was performed to explore the expression of two most commonly studied chemokines CX3CL1 and CCL2 and assess their associations with clinical severity in LDH patients with sciatic pain.

Methods: The soft tissues around nerve root (STANR), annulus fibrosus (AF), and nucleus pulposus (NP) biopsies were obtained from $36 \mathrm{LDH}$ patients with chronic sciatic pain and 10 scoliosis patients (painless controls). The serum and local expressions of CX3CL1 and CCL2 were determined using enzyme-linked immunosorbent assay and Western blot analysis, respectively. The visual analog scale (VAS) scores for low back pain and lower extremity pain and Japanese Orthopaedic Association (JOA) scores were recorded on the day of hospital admission to evaluate the clinical severity. LDH patients with sciatic pain were divided into severe pain (SP) group (VAS $\geq 7 ; n=18$ ) and mild-to-moderate pain (M-MP) group (VAS <7; n=18) for lower extremity pain.

Results: Local expressions instead of CX3CL1 and CCL2 in STANR, AF, and NP were significantly higher in the SP group than in M-MP compared with scoliosis painless group. Expressions of both CX3CL1 and CCL2 in STANR and AF were positively correlated with VAS scores for lower extremity and for low back pain, respectively. In addition, CX3CL1 and CCL2 expressions in STANR were negatively associated with JOA scores. There were no significant differences of serum CX3CL1 and CCL2 levels among SP group, M-MP group, and scoliosis painless group. Conclusion: Both CX3CL1 and CCL2 may play important roles in maintaining pain in LDH patients. Local blockade of CX3CL1 and CCL2 in LDH patients with persistent pain deserves further intensive study.

Keywords: lumbar disk herniation, sciatic pain, CX3CL1, CCL2

\section{Introduction}

Lumbar disk herniation (LDH) is one of the most common spinal degenerative disorders that frequently affects the spine in young and middle-aged adults. ${ }^{1}$ The clinical consequences of LDH physiology are spinal pain and stiffness, prolapse with nerve root pain, and stenosis with spinal claudication. ${ }^{2}$ Pain is the most common complaint in LDH patients. Pain in LDH is present either local to the disordered disk or in the lower limb with associated paresthesiae caused by root compression and spinal stenosis. ${ }^{3}$

In the past, pain in patients with disk diseases including LDH has long been attributed to the pressure put on the sciatic nerve root by the herniated disk. However,
Correspondence: $\mathrm{Xi}$-Yang Wang Department of Spine Surgery, Xiangya Hospital, Central South University, No. 87 Xiangya Road, Changsha, Hunan 4I0008, People's Republic of China $\mathrm{Tel}+8673184328888$

Email wxyorthoxy@I63.com 
advances in basic and clinical study have clarified the fact that production of pain in LDH is not only due to mechanic pressure but is also due to a significant contribution by chemical factors. ${ }^{4}$ This conclusion is based on the following clinical observations. 1) Disk surgery does not always release pain; 2) large disk herniations are not always symptomatic; 3) severe pain (SP) may be present in patients without imaging evidence of nerve root compression; 4) the severity of symptoms and neurological signs is not well related to the size of the disk herniation; and 5) conservative therapy is often effective. ${ }^{5-8}$

Recent basic studies have strengthened the potential role for autoimmunity in the progression of LDH. The diskderived cells are able to secrete pro-inflammatory cytokines without inflammatory cells, supporting the hypothesis that disk-derived cells have the capacity of initiating as well as amplifying the inflammatory process. ${ }^{9}$ Furthermore, infiltration and activation of various immune cells contribute to amplification of an inflammatory response and the release of neurotrophic factors that aggravate neoinnervation of the disk or nerve sensitization of spinal nerves. ${ }^{10,11}$

Chemokines constitute of a group of pro-inflammatory cytokines with potent chemotactic activities for leukocytes both in vitro and in vivo. ${ }^{12}$ Chemokines are classified into four groups ( $\mathrm{CC}, \mathrm{XC}, \mathrm{CXC}$, and $\mathrm{CX} 3 \mathrm{C}$ ) based on the number and position of conserved cysteine residues in their amino acid structure, and their receptors are accordingly designated as CCR, XCR, CXCR, or CX3CR. ${ }^{13}$ The chemokines act both at the initial and later stages of inflammatory response and draw inflammatory cells from blood into tissues. ${ }^{14}$

Among the chemokines, the $\mathrm{CX} 3 \mathrm{C}$ and $\mathrm{CC}$ subfamilies are most widely studied. Specifically, CX3CL1 (C-X3-C motif ligand 1), also named as fractalkine, is structurally unique among the family of chemokines and their receptors and is expressed both in the central nervous system and peripheral nerves, as well as in endothelial cells and lymphocytes. CCL2 (C-C motif ligand 2), also known as monocytes chemoattractant protein-1, is a chemokine that has been demonstrated to play a critical role in chronic pain facilitation via CCR2. CCL2 and CX3CL1 signaling through CCR2 and CX3CR1 have been shown to be key factors in the recruitment of inflammation cells to tissue lesions or sites of inflammation, ${ }^{15}$ and so are key factors in persistence of neuropathic pain. ${ }^{16,17}$

A previous study has shown that chemokine (including CX3CL1 and CCL2) production by herniated intervertebral disk tissue is the reason for the generation and maintenance of surrounding inflammation. ${ }^{18}$ Park et al ${ }^{19}$ found that
CX3CL1 immunoreactivity gradually increased in laminae II and III of the ipsilateral dorsal horn by using a nucleus pulposus (NP) implantation-induced model of LDH. One recent study showed that CCL2/CCR2 signaling in the dorsal root ganglion and spinal cord plays a significant role in the maintenance of neuropathic pain in a rat model of $\mathrm{LDH} .^{20}$

All these works implicated that CCL2 and CX3CL1 may both play important roles in pain maintenance and aggravation in LDH patients. To our knowledge, most of the studies in this area were carried out on animal models, and there were no studies available illustrating the relationship between systemic and local chemokine expressions and clinical severity in LDH patients. Therefore, we aimed to investigate the potential mechanism from the biochemical aspects by exploring expressions of CCL2 and CX3CL1 in LDH patients and by assessing their association with clinical severity.

\section{Methods}

\section{Study patients and clinical assessment}

This study was approved by the Ethics Committee at Chenzhou No. 1 People's Hospital, Hunan, People's Republic of China. Thirty-six patients diagnosed as having LDH with sciatic pain were enrolled at the Department of Spinal Orthopedics. The clinical inclusion criteria are as follows: 1) low back pain with radiation to the lower limb; 2) radicular pain along a specific dermatome; 3 ) nerve root tension signs like straight leg raising test; and 4) presence of neurological symptoms and signs. Diagnosis of LDH was further confirmed using magnetic resonance imaging. Also, ten scoliosis patients without signs of pain or degenerative disk disease were selected as controls. Written informed consent was obtained from all the patients (or from their parents if they were under the age of 16 years).

The soft tissues around nerve root (STANR), annulus fibrosus (AF), and NP biopsies were obtained intraoperatively from $36 \mathrm{LDH}$ patients with chronic sciatic pain during diskectomy and were obtained from ten scoliosis patients (painless controls) during corrective orthopedic surgery.

\section{Assessment of serum CX3CLI and CCL2 levels}

About $10 \mathrm{~mL}$ of blood sample was collected from each patient in the morning and then centrifuged at 3,000 rpm for $10 \mathrm{~min}$. The obtained serum was then stored at $-80^{\circ} \mathrm{C}$ until use. Serum CX3CL1 and CCL2 levels were measured using the enzyme-linked immunosorbent assay technique (Santa Cruz Biotechnologies, Inc., Santa Cruz, CA, USA). Intra-assay 
coefficients of variability were $4.1 \%$ and $4.4 \%$, respectively, and the interassay coefficients of variability were less than $6 \%$.

\section{Local CX3CLI and CCL2 protein expressions explored using Western blot analysis}

Tissue samples from STANR, AF, and NP were rapidly removed and stored at $-80^{\circ} \mathrm{C}$ until use. All tissue samples were homogenized in lysis buffer containing phenylmethane sulfonyl fluoride and $0.02 \%$ protease inhibitor cocktail. The homogenates were centrifuged at $14,000 \times g$ for $15 \mathrm{~min}$ at $4^{\circ} \mathrm{C}$. Equivalent amounts of protein $(40 \mu \mathrm{g})$ were separated using 10\% sodium dodecyl sulfate-polyacrylamide gel electrophoresis and transferred onto a polyvinylidene difluoride membrane. Membranes were blocked with 5\% milk for $1 \mathrm{~h}$ at room temperature and incubated with respective primary antibodies anti-CX3CL1 (1:1,000; Santa Cruz Biotechnologies, Inc) and anti-CCL2 (1:4,000; Abcam, Cambridge, UK) overnight at $4^{\circ} \mathrm{C}$. The membranes were incubated for $1 \mathrm{~h}$ with horseradish peroxidase-conjugated secondary antibody (1:5,000; Santa Cruz Biotechnologies, Inc). Bands were finally revealed using an enhanced chemiluminescence kit (Kodak, Rochester, NY, USA). The monoclonal antibody against $\beta$-actin (1:10,000; Sigma-Aldrich Co., St Louis, MO, USA) was used as a loading control. Bands area/intensity for all proteins were measured using Image $J$ software (Wayne Rasband; National Institutes of Health, Bethesda, MD, USA). All the samples were routinely analyzed using Western blot repeated for at least three times, and the obtained results were averaged and calculated.

\section{Pain and function assessment}

The visual analog scale (VAS) was administered to evaluate the degree of pain, ranging from 0 to 10 , where 0 indicates complete satisfaction or no pain at all, whereas 10 indicates complete dissatisfaction or maximum pain. Here, we recorded VAS scores for both lower extremity pain and low back pain.
LDH patients with sciatic pain were divided into SP group (VAS $\geq 7 ; n=18$ ) and mild-to-moderate pain (M-MP) group (VAS $<7 ; n=18$ ) for lower extremity pain. Japanese Orthopedic Association (JOA) scores were utilized to assess the comprehensive functional status in patients. The JOA score system is a widely used clinical evaluation scoring system for low back pain syndrome including subjective symptoms, clinical signs, restriction of activities of daily living, and urinary bladder function, ranging from 0 to 29 points, where higher scores represent less pain and better function and lower scores indicate SP and worse function. The JOA score system is a validated instrument designed specifically for the assessment of patients with low back pain and worse function. JOA score is widely used based on the clinical evidence and experience. ${ }^{21}$

\section{Statistical analysis}

Statistical analysis was performed using the Statistical Package for Social Sciences 21.0 statistical software (IBM, Armonk, NY, USA). Data were presented as mean \pm standard deviation or median (interquartile range). Data normality was analyzed using the Kolmogorov-Smirnov test. The one-way analysis of variance or Kruskal-Wallis test was applied to test statistically significant differences in protein expression between SP and M-MP LDH patient groups as well as the scoliosis control group, followed by a Dunn's post hoc test. The Spearman's or Pearson's correlation analysis was used to examine the association of CX3CL1 or CCL2 level with VAS as well as JOA scores. Probability values or differences $<0.05$ were considered to be statistically significant.

\section{Results}

\section{Baseline clinical characteristics}

With the exception of significant difference in age $(P<0.001)$, no significant differences were observed in indices of sex ratio, disease duration, body mass index, and serum CX3CL1 and CCL2 levels among the three groups. The basic clinical and laboratory characteristics are listed in Table 1.

Table I Baseline clinical characteristics

\begin{tabular}{|c|c|c|c|c|}
\hline & SP & M-MP & Con & $P$-value \\
\hline $\mathrm{N}$ & 18 & 18 & 10 & NS \\
\hline Sex (female/male) & $7 / 11$ & $9 / 9$ & $5 / 5$ & NS \\
\hline Age (years) & $34.2 \pm 5.8$ & $33.3 \pm 6.8$ & $22.1 \pm 4.1$ & $<0.001$ \\
\hline Disease duration (months) & $4.5(I-22)$ & $5.2(2-18)$ & $72(36-96)$ & - \\
\hline $\mathrm{BMI}\left(\mathrm{kg} / \mathrm{m}^{2}\right)$ & $22.69(18.15-24.53)$ & $23.12(18.33-25.01)$ & $21.58(17.68-24.72)$ & NS \\
\hline Serum CX3CLI levels (pg/mL) & $120.8 \pm 14.4$ & $112.8 \pm 15.5$ & $113.6 \pm 11.4$ & NS \\
\hline Serum CCL2 levels (pg/mL) & $94.2 \pm 10.5$ & $90.7 \pm 10.8$ & $85.1 \pm 12.9$ & NS \\
\hline
\end{tabular}

Abbreviations: SP, severe pain; M-MP, mild-to-moderate pain; Con, scoliosis painless control; NS, not significant; BMI, body mass index. 


\section{Serum and local CX3CLI and CCL2} expressions in LDH patients and controls

According to the extent of pain, LDH patients with sciatic pain were divided into SP group (VAS $\geq 7 ; n=18$ ) and M-MP group (VAS $<7 ; n=18$ ). In addition, ten scoliosis painless controls were selected as nonpainful controls. No significant differences were found both in serum CX3CL1 and CCL2 levels among SP LDH patients, M-MP LDH patients, and scoliosis painless controls (CX3CL1: $120.8 \pm 14.4 \mathrm{pg} / \mathrm{mL}$ vs $112.8 \pm 15.5 \mathrm{pg} / \mathrm{mL}$ vs $113.6 \pm 11.4 \mathrm{pg} / \mathrm{mL}, P>0.05$; CCL2: $94.2 \pm 10.5 \mathrm{pg} / \mathrm{mL}$ vs $90.7 \pm 10.8 \mathrm{pg} / \mathrm{mL}$ vs $85.1 \pm 12.9$ $\mathrm{pg} / \mathrm{mL}$ ) (Table 1; Figure S1A and B). Both STANR, NP, and AF CX3CL1 and CCL2 protein expressions in SP and M-MP groups were significantly higher than those in the scoliosis painless control group (All $P<0.05$; Figures 1 and $2 \mathrm{~A}-\mathrm{E})$. We further compared the local CX3CL1 and CCL2 expressions between SP and M-MP groups. We found only the STANR CX3CL1 but not CCL2 expressions were significantly higher in SP group compared with M-MP group $(P=0.02$; Figure $2 \mathrm{~A})$. However, interestingly, NP but not STANR CCL2 expression was higher in SP group compared with M-MP group ( $P=0.04$; Figures 1 and $2 \mathrm{E}$ ).

\section{Association of local CX3CLI and CCL2 protein expression levels with VAS and JOA scores}

The associations of CX3CL1 and CCL2 protein expression levels in STANR, AF, and NP with VAS scores as well as
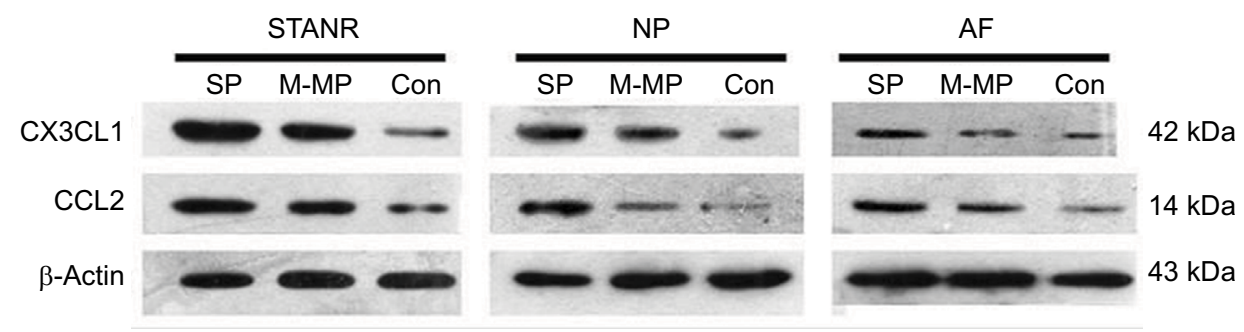

Figure I CX3CLI and CCL2 band expressions were determined using Western blot analysis among three groups.

Notes: Expression levels were normalized to $\beta$-actin as house-keeper factor.

Abbreviations: STANR, soft tissues around nerve root; SP, severe pain; M-MP, mild-to-moderate pain; Con, scoliosis painless control; AF, annulus fibrosus; NP, nucleus pulposus.
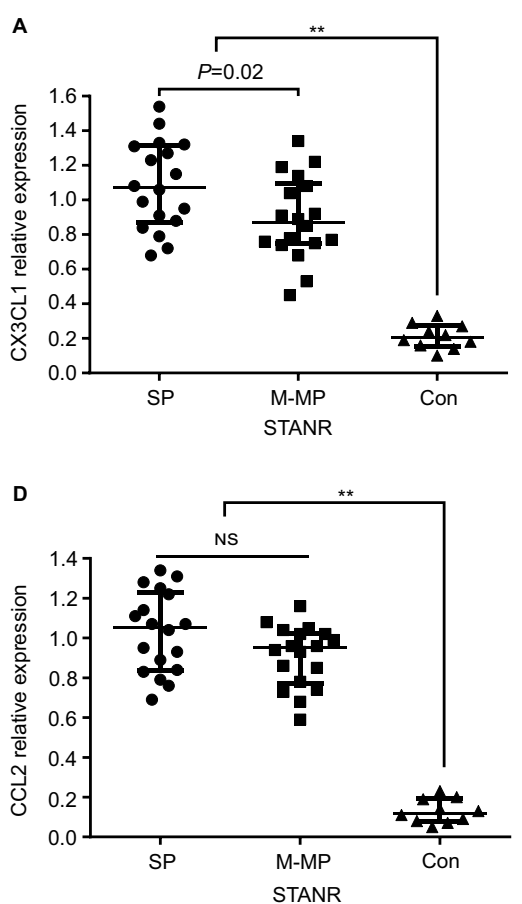
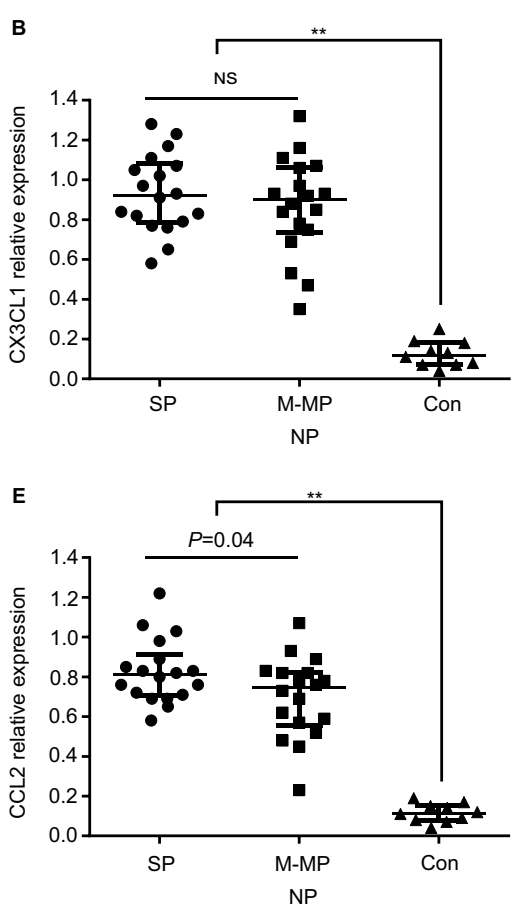
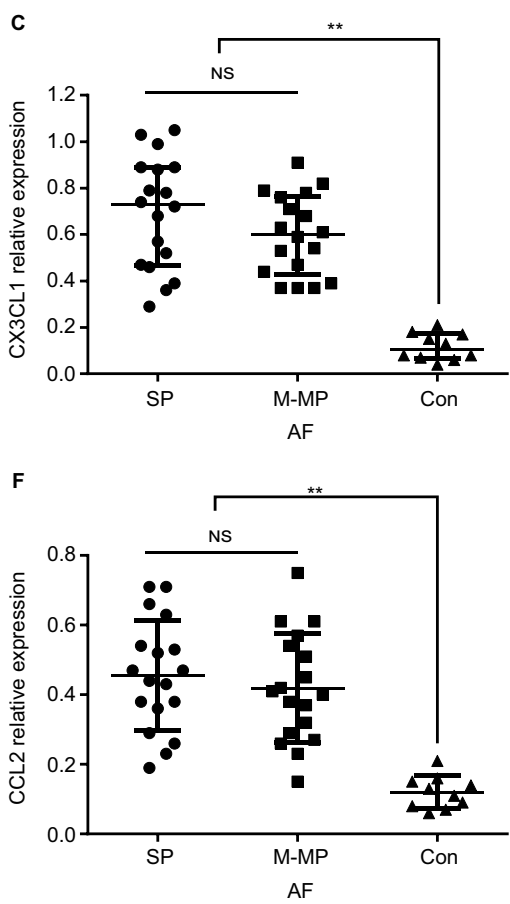

Figure 2 Quantification analysis of $C X 3 C L 1$ and $C C L 2$ protein expressions among three groups.

Notes: Expression levels were normalized to $\beta$-actin as house-keeper factor and are represented as time-fold increase compared with controls. (A-C) CX3CLI protein expressions among three groups in STANR (A), NP (B), and AF (C). (D-F) CCL2 protein expressions among three groups in STANR (D), NP (E), and AF (F). **P<0.01. Abbreviations: SP, severe pain; M-MP, mild-to-moderate pain; Con, scoliosis painless control; NS, not significant; AF, annulus fibrosus; NP, nucleus pulposus; STANR, soft tissues around nerve root. 
JOA scores of patients with LDH were explored. Correlation analysis showed that only STANR CX3CL1 and CCL2 protein expression levels in patients were positively correlated with VAS (CX3CL1: $r=0.393, P=0.018$ and CCL2: $r=0.360, P=0.03$ ) for lower extremity pain (Figure $3 \mathrm{~A}-\mathrm{F}$ ) and negatively related to JOA scores (CX3CL1: $r=-0.342$,
$P=0.04$ and CCL2: $r=-0.375, P=0.02$; Figure $4 \mathrm{~A}-\mathrm{F})$. In addition, CX3CL1 and CCL2 protein expression levels in AF (Figure 5C and F) instead of STANR (Figure 5A and D) were also positively correlated with VAS for low back pain (CX3CL1: $r=0.349, P=0.04$ and CCL2: $r=0.365, P=0.03$ ). However, correlations of CX3CL1 and CCL2 protein
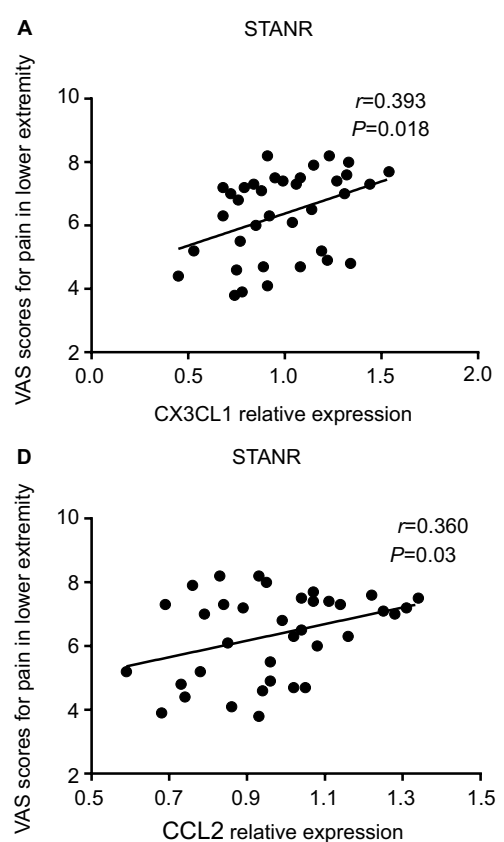
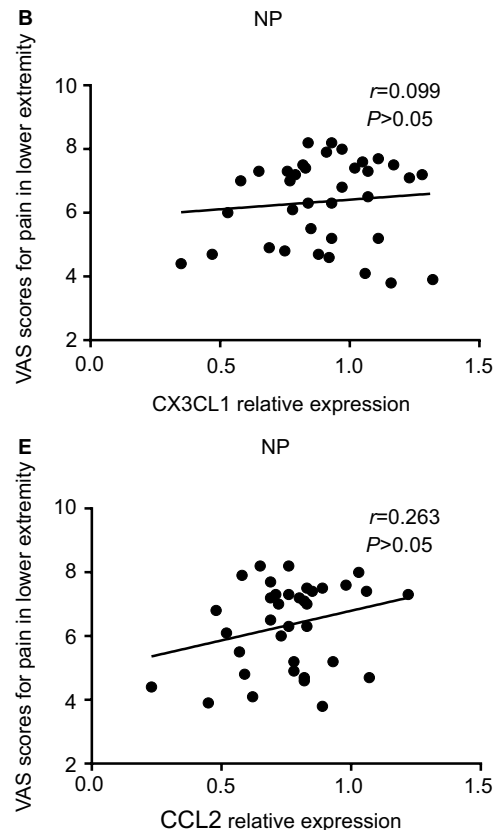
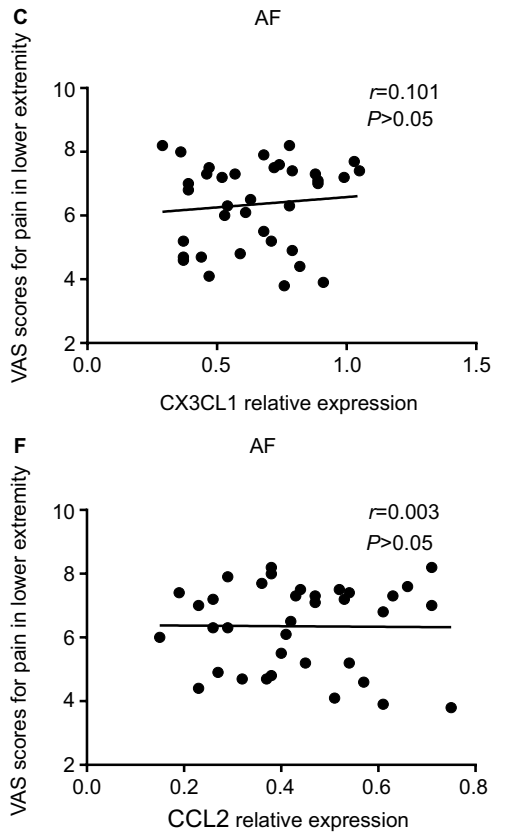

Figure 3 Correlation of local CX3CLI and CCL2 expressions with VAS scores (in centimeters) for pain in lower extremity.

Notes: (A-C) Correlation of CX3CLI protein expressions with VAS scores for pain in lower extremity in STANR (A), NP (B), and AF (C), respectively. (D-F) Correlation of CCL2 protein expressions with VAS scores for pain in lower extremity in STANR (D), NP (E), and AF (F), respectively.

Abbreviations: AF, annulus fibrosus; NP, nucleus pulposus; VAS, visual analog scale; STANR, soft tissues around nerve root.
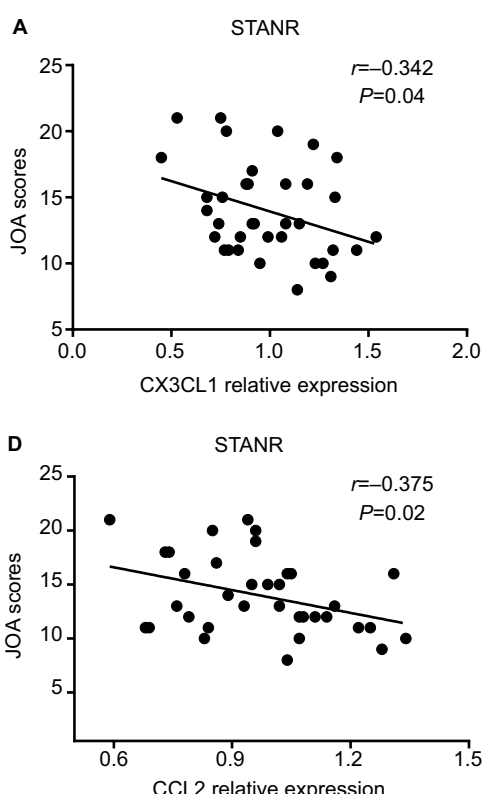

B NP
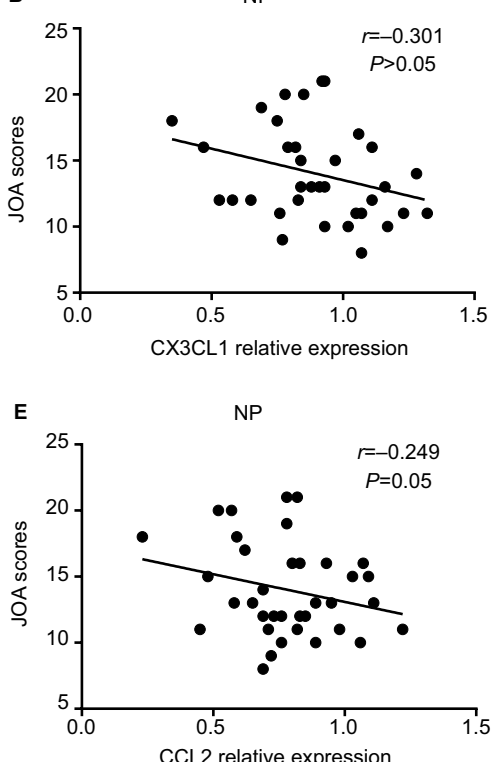
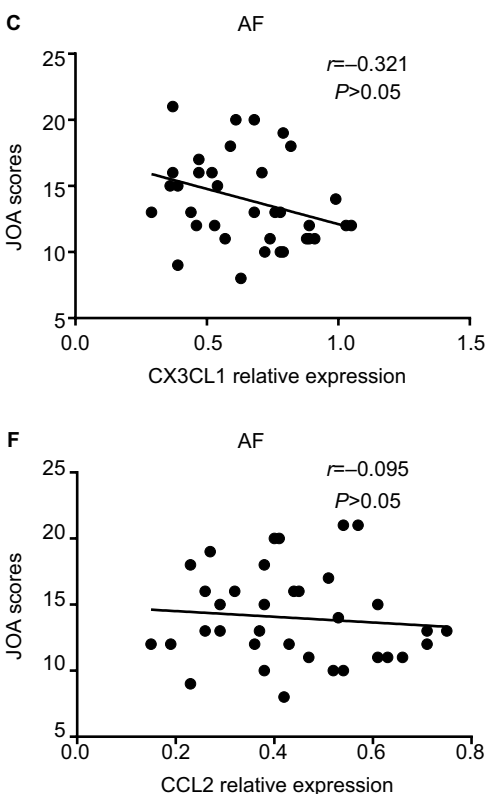

Figure 4 Correlation of local CX3CLI (A-C) and CCL2 (D-F) expressions with JOA scores.

Notes: (A-C) Correlation of CX3CLI protein expressions with JOA scores in STANR (A), NP (B), and AF (C), respectively. (D-F) Correlation of CCL2 protein expressions with JOA scores in STANR (D), NP (E), and AF (F), respectively.

Abbreviations: AF, annulus fibrosus; NP, nucleus pulposus; JOA, Japanese Orthopedic Association; STANR, soft tissues around nerve root. 

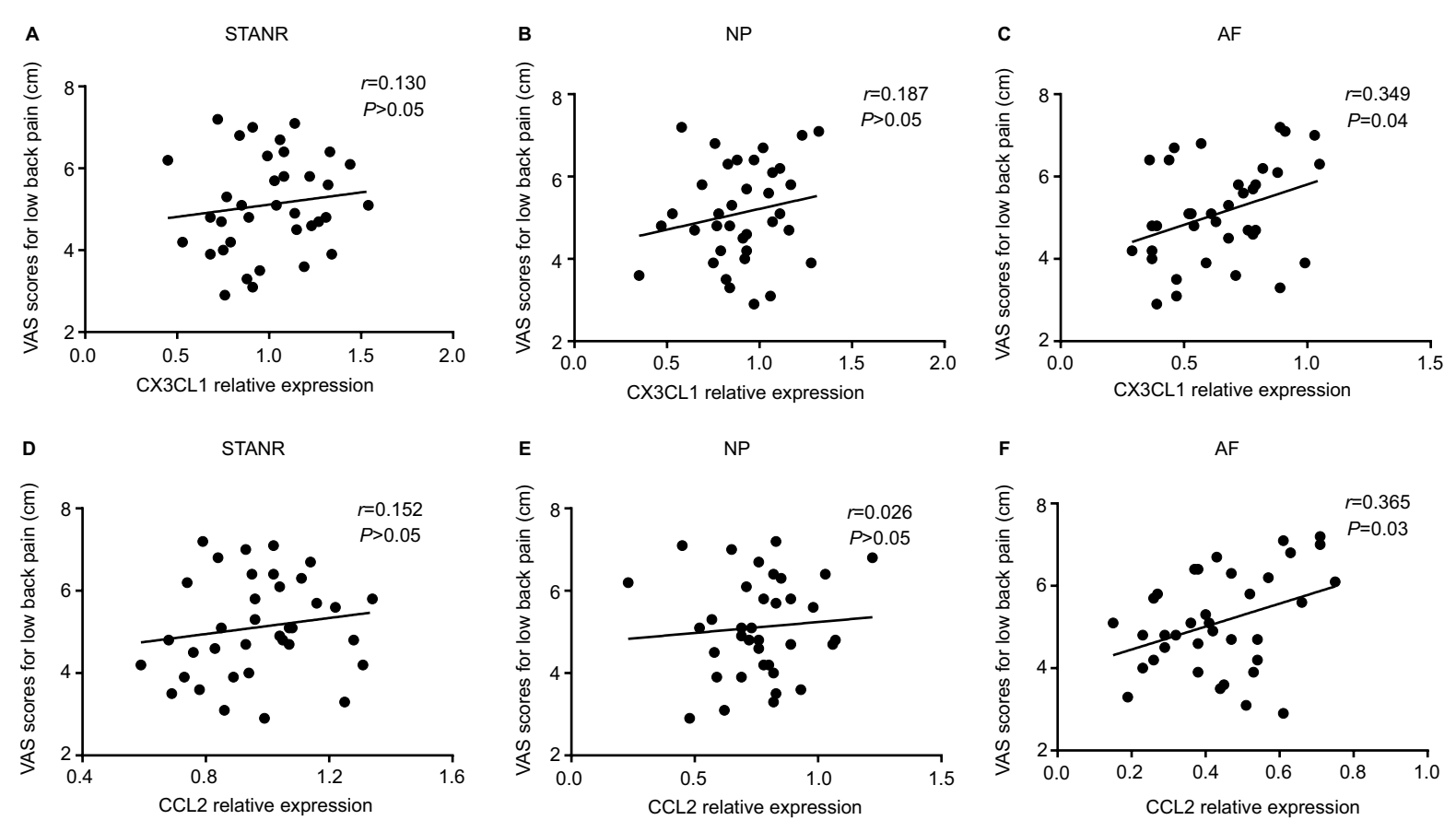

Figure 5 Correlation of local CX3CLI and CCL2 expressions with VAS scores (in centimeters) for low back pain. Notes: (A-C) Correlation of CX3CLI protein expressions with VAS scores for low back pain in STANR (A), NP (B), and AF (C), respectively. (D-F) Correlation of CCL2 protein expressions with VAS scores for low back pain in STANR (D), NP (E), and AF (F), respectively.

Abbreviations: SP, severe pain; STANR, soft tissues around nerve root; AF, annulus fibrosus; NP, nucleus pulposus; VAS, visual analog scale.

expression levels in NP with VAS and JOA did not reach significant differences (all $P>0.05$; Figure 3B and E; Figure 5B and E; Figure 4B and E).

\section{Discussion}

This study was performed to examine the local and circulating levels of the most widely studied chemokines CX3CL1 and CCL2 in LDH patients with radicular pain. To our knowledge, this is the first time that we explored CX3CL1 and CCL2 expressions in $\mathrm{LDH}$ patients. Our findings demonstrated that the local expressions instead of serum levels of CX3CL1 and CCL2 are significantly higher in LDH patients compared with controls. In addition, we also investigated the relationship of the two chemokines and disease severity defined by the degree of pain and functional disability. We observed that both CX3CL1 and CCL2 expressions are significantly associated with more serious pain and worse function. These findings implicated that local CX3CL1 and CCL2 play potential roles in the progression and aggravation of the disease.

$\mathrm{LDH}$, which is manifested by low back pain or sciatica, is one of the common causes of disability in the community. The reported lifetime occurrence of $\mathrm{LDH}$ achieves as high as $40 \%{ }^{22}$ In LDH, NP material exits the disk space area through the AF, causing mild compression on the spinal nerves and resulting in neurological dysfunction including pain, sensory deficits, and weakness in the low back and leg. ${ }^{23}$ In recent years, a growing number of works have identified the role of inflammation and immune activation in the pathogenesis of lumbar disk disease. Cytokines including interleukin (IL)-1 and tumor necrosis factor (TNF)- $\alpha$ are found to be elevated in LDH patients with or without sciatic pain. ${ }^{24,25}$ It has also been proved that LDH patients with chronic pain had higher levels of TNF- $\alpha$, IL-1, and IL- 6 than controls, ${ }^{26,27}$ thus supporting the role of inflammation on aggravation and maintenance of pain.

As one important family of cytokines, chemokines in recent years have been shown to play a pivotal role in the induction and maintenance of inflammation and chronic pain. ${ }^{28}$ The best known chemokines in pain are CX3CL1 and CCL2, which signal through their designated receptors. Local expressions of CX3CL1 and CCL2 in STANR, AF, and NP were all significantly higher in the SP group than in M-MP compared with scoliosis painless group. However, we found that serum CX3CL1 and CCL2 levels between LDH patients and control subjects did not reach significant difference, implicating that inflammation or pain in LDH is more like a local process rather than systemic activity. We further found both expressions of CX3CL1 and CCL2 in STANR and AF were positively correlated with VAS scores for lower extremity pain and low back pain, respectively. However, only the local expressions of both the chemokines in AF negatively associated with JOA scores. This may be attributed to the fact that pain in lower extremity and low back were generated by different nerves. The former is generated by sinuvertebral nerve and the latter is produced by sciatic nerve. The sciatic 
nerve has much more fibers than the sinuvertebral nerve and is more sensitive to pain. These results above both implicated that CX3CL1 and CCL2 may play important roles in the nerve root inflammation and neuropathic pain.

CX3CL1/CX3CR1 mediates neuron-microglial communication in chronic pain states and is therefore key to the development of neuropathic pain. ${ }^{29}$ In addition, peripheral inflammation-induced hypernociception is accompanied and mediated by satellite glial cells activation. Souza et $\mathrm{al}^{30}$ found that CX3CL1/CX3CR1 signaling in the dorsal root ganglion plays a key role in the peripheral inflammation and the activation of satellite glial cells, and they conclude this process is crucial to the induction/maintenance of inflammatory pain. Oh et $\mathrm{a}^{31}$ evaluated the expression of CX3CL1 and CX3CR1 in patients with cervical and LDH to clarify their roles in the disk degeneration. They found greater CX3CL1/CX3CR1 mRNA/CX3CR1-positive cells expression in cervical herniated NP patients than in lumbar herniated NP patients using reverse transcription polymerase chain reaction and histologic and immunohistochemical analysis. However, references of symptoms including pain and function were not recorded. Therefore, we speculate that CX3CL1 and CX3CR1 would exhibit higher expressions when pain symptoms are present.

CCL2 is another well-known chemokine amplifying and maintaining inflammation through chemokine-cytokine networks following the recruitment of circulating leukocytes. Zhu et $\mathrm{al}^{32}$ found that LDH could induce overexpression of CCL2 in NP cells. And, elevated CCL2 expression has been also detected in LDH animal models and human degenerated disks. ${ }^{33}$ Several studies have also shown that CCL2 is involved in the activation of spinal microglia associated with neuropathic pain, ${ }^{34,35}$ which can result in central sensitization by the release of a series of modulators, which enhances pain states further.

Low back pain in LDH patients is believed to originate from internal disk disruptions and is mainly mediated by the sinuvertebral nerve. ${ }^{36}$ Here, we also found that elevated CX3CL1 and CCL2 expressions in AF instead of NP were positively associated with low back pain. This may be attributed to free sinuvertebral nerve endings that are mainly distributed in the superficial area of AF, but not in NP and deeper zones of AF. ${ }^{37}$

Some limitations should be taken into account in the present study. First, this study was performed only in Chinese people, and the sample size enrolled was not large enough, thus leading to limited statistical power. Therefore, multicenter studies with a large sample size are further needed to identify the present results. Second, we only focused the investigation of CX3CL1 and CCL2, examination of other inflammatory markers such as IL-1 and TNF- $\alpha$ may be more powerful and meaningful to illustrate their roles in LDH inflammation. Finally, we were not able to examine the systemic and local CX3CL1 and CCL2 expression levels due to ethical reasons, failing to get the information about the alternation of CX3CL1 and CCL2 expression following symptomatic relief.

\section{Conclusion}

In summary, local instead of serum CX3CL1 and CCL2 protein expressions in $\mathrm{LDH}$ patients with sciatic pain were higher than in scoliosis painless control. In addition, local expressions of CX3CL1 and CCL2 in STANR were positively correlated with SP and worse function. Local CX3CL1 and CCL2 protein expressions may reflect clinical severity in LDH patients with sciatic pain. Local expressions of CX3CL1 and CCL2 have the potential to serve as targets for adjunctive treatment of LDH combined with other therapies, but the clinical applications need intensive study in the future.

\section{Disclosure}

The authors report no conflicts of interest in this work.

\section{References}

1. Benoist $M$. The natural history of lumbar disc herniation and radiculopathy. Joint Bone Spine. 2002;69:155-160.

2. Latka D, Miekisiak G, Jarmuzek P, Lachowski M, Kaczmarczyk J. Treatment of lumbar disc herniation with radiculopathy. Clinical practice guidelines endorsed by The Polish Society of Spinal Surgery. Neurol Neurochir Pol. 2016;50:101-108.

3. Li Y, Fredrickson V, Resnick DK. How should we grade lumbar disc herniation and nerve root compression? a systematic review. Clin Orthop Relat Res. 2015;473:1896-1902

4. Di Martino A, Merlini L, Faldini C. Autoimmunity in intervertebral disc herniation: from bench to bedside. Expert Opin Ther Targets. 2013;17:1461-1470.

5. Boden SD, Davis DO, Dina TS, Patronas NJ, Wiesel SW. Abnormal magnetic resonance scans of the lumbar spine in asymptomatic subjects: a prospective investigation. J Bone Jt Surg. 1990;72A:403-408.

6. Ohnmeiss DD, Vanharanta H, Ekholm J. Degree of disc disruption and lower extremity pain. Spine. 1997;22:600-605.

7. Bejia I, Younes M, Zrour S, Touzi M, Bergaoui N. Factors predicting outcomes of mechanical sciatica: a review of 1092 cases. Joint Bone Spine. 2004;71:567-571.

8. Valls I, Saraux A, Goupille P, Khoreichi A, Baron D, Le Goff P. Factors predicting radical treatment after in-hospital conservative management of disk-related sciatica. Joint Bone Spine. 2001;68:50-58.

9. Olmarker K, Blomquist J, Stromberg J, Nannmark U, Thomsen P, Rydevik B. Inflammatogenic properties of nucleus pulposus. Spine (Phila Pa 1976). 1995;20:665-669

10. Risbud MV, Shapiro IM. Role of cytokines in intervertebral disc degeneration: pain and disc content. Nat Rev Rheumatol. 2014;10:44-56.

11. Krock E, Rosenzweig DH, Chabot-Dore AJ, et al. Painful, degenerating intervertebral discs up-regulate neurite sprouting and CGRP through nociceptive factors. J Cell Mol Med. 2014;18:1213-1225.

12. Colobran R, Pujol-Borrell R, Armengol MP, Juan M. The chemokine network. I. How the genomic organization of chemokines contains clues for deciphering their functional complexity. Clin Exp Immunol. 2007;148:208-217. 
13. Zlotnik A, Yoshie O. The chemokine superfamily revisited. Immunity. 2012;36:705-716

14. Muñoz LM, Holgado BL, Martınez-A C, Rodríguez-Frade JM, Mellado M. Chemokine receptor oligomerization: a further step toward chemokine function. Immunol Lett. 2012;145:23-29

15. Luster AD. Chemokines-chemotactic cytokines that mediate inflammation. N Engl J Med. 1998;338:436-445.

16. Biber K, Boddeke E. Neuronal CC chemokines: the distinct roles of CCL21 and CCL2 in neuropathic pain. Front Cell Neurosci. 2014; $8: 210$.

17. Clark AK, Malcangio M. Fractalkine/CX3CR1 signaling during neuropathic pain. Front Cell Neurosci. 2014;8:121.

18. Burke JG, Watson RW, McCormack D, Dowling FE, Walsh MG, Fitzpatrick JM. Spontaneous production of monocyte chemoattractant protein-1 and interleukin-8 by the human lumbar intervertebral disc. Spine (Phila Pa 1976). 2002;27:1402-1407.

19. Park HW, Ahn SH, Kim SJ, et al. Changes in spinal cord expression of fractalkine and its receptor in a rat model of disc herniation by autologous nucleus pulposus. Spine (Phila Pa 1976). 2011;36:E753-E760.

20. Zhu X, Cao S, Zhu MD, Liu JQ, Chen JJ, Gao YJ. Contribution of chemokine CCL2/CCR2 signaling in the dorsal root ganglion and spinal cord to the maintenance of neuropathic pain in a rat model of lumbar disc herniation. J Pain. 2014;15:516-526.

21. Ohtori S, Ito T, Yamashita M, et al. Evaluation of low back pain using the Japanese Orthopaedic Association Back Pain Evaluation Questionnaire for lumbar spinal disease in a multicenter study: differences in scores based on age, sex, and type of disease. J Orthop Sci. 2010; 15:86-91.

22. Frymoyer JW, Pope MH, Clements JH, Wilder DG, MacPherson B, Ashikaga T. Risk factors in low-back pain. An epidemiological survey. J Bone Joint Surg. 1983;65:213-218.

23. Chan WC, Sze KL, Samartzis D, Leung VY, Chan D. Structure and biology of the intervertebral disk in health and disease. Orthop Clin North Am. 2011;42:447-464

24. Studer RK, Vo N, Sowa G, Ondeck C, Kang J. Human nucleus pulposus cells react to IL-6: independent actions and amplification of response to IL-1 and TNF- $\alpha$. Spine (Phila Pa 1976). 2011;36:593-599.
25. Williams NH, Lewis R, Din NU, et al. A systematic review and metaanalysis of biological treatments targeting tumour necrosis factor $\alpha$ for sciatica. Eur Spine J. 2013;22:1921-1935.

26. Genevay S, Finckh A, Payer M, et al. Elevated levels of tumor necrosis factor-alpha in periradicular fat tissue in patients with radiculopathy from herniated disc. Spine (Phila Pa 1976). 2008;33:2041-2046

27. Andrade P, Hoogland G, Garcia MA, Steinbusch HW, Daemen MA, Visser-Vandewalle V. Elevated IL-1 $\beta$ and IL-6 levels in lumbar herniated discs in patients with sciatic pain. Eur Spine J. 2013;22:714-720.

28. Kiguchi N, Kobayashi Y, Kishioka S. Chemokines and cytokines in neuroinflammation leading to neuropathic pain. Curr Opin Pharmacol. 2012;12:55-61.

29. Clark AK, Staniland AA, Malcangio M. Fractalkine/CX3CR1 signalling in chronic pain and inflammation. Curr Pharm Biotechnol. 2011;12:1707-1714.

30. Souza GR, Talbot J, Lotufo CM, Cunha FQ, Cunha TM, Ferreira SH. Fractalkine mediates inflammatory pain through activation of satellite glial cells. Proc Natl Acad Sci USA. 2013;110:11193-1118.

31. Oh IS, Suh DW, Park SR, Ha KY. Fractalkine receptor chemokine (CX3CR1) influences on cervical and lumbar disc herniation. Indian $J$ Orthop. 2015;49:239-244.

32. Zhu Z, Huang P, Chong Y, et al. Nucleus pulposus cells derived IGF-1 and MCP-1 enhance osteoclastogenesis and vertebrae disruption in lumbar disc herniation. Int J Clin Exp Pathol. 2014;7:8520-8531.

33. Harao H, Komori H, Okawa A, Murakami S, Muneta T, Shinomiya K. Sequential dynamics of monocyte chemotactin protein-1 expression in herniated nucleus pulposus resorption. J Orthop Res. 1997;15:734-741.

34. Thacker MA, Clark AK, Bishop T, et al. CCL2 is a key mediator of microglia activation in neuropathic pain states. Eur J Pain. 2009;13:263-272.

35. Zhang J, Shi XQ, Echeverry S, Mogil JS, De Koninck Y, Rivest S. Expression of CCR 2 in both resident and bone marrow-derived microglia plays a critical role in neuropathic pain. J Neurosci. 2007;27:12396-12406.

36. Schliessbach J, Siegenthaler A, Heini P, Bogduk N, Curatolo M. Blockade of the sinuvertebral nerve for the diagnosis of lumbar diskogenic pain: an exploratory study. Anesth Analg. 2010;111:204-206.

37. Takahashi K, Aoki Y, Ohtori S. Resolving discogenic pain. Eur Spine J. 2008;4:428-431. 


\section{Supplementary material}

A

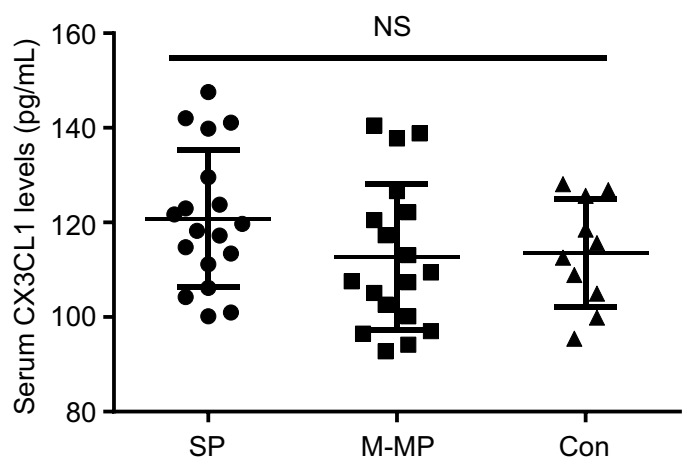

B

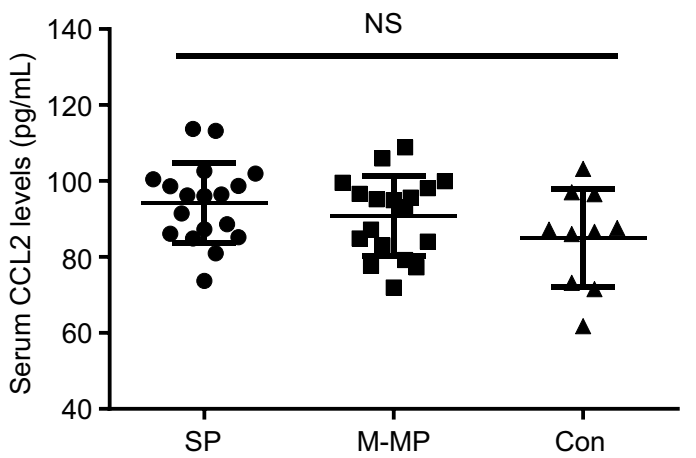

Figure SI Investigation of serum CX3CLI (A) and CCL2 (B) among three groups.

Abbreviations: SP, severe pain; M-MP, mild-to-moderate pain; Con, scoliosis painless control; NS, not significant.

The Journal of Pain Research is an international, peer reviewed, open access, online journal that welcomes laboratory and clinical findings in the fields of pain research and the prevention and management of pain. Original research, reviews, symposium reports, hypothesis formation and commentaries are all considered for publication.

\section{Dovepress}

The manuscript management system is completely online and includes a very quick and fair peer-review system, which is all easy to use. Visit http://www.dovepress.com/testimonials.php to read real quotes from published authors. 